MATEC Web of Conferences 22,05018 (2015)

DOI: $10.1051 /$ matec conf/ 20152205018

(C) Owned by the authors, published by EDP Sciences, 2015

\title{
Effects of Physical Exercise and Sedentary Behavior on the Chronic Diseases Morbidity in a Chinese Adult Population
}

\author{
Qing Wei*, Wenbin Wang \& Kaiye Gao \\ Donlinks School of Economic and Management, University of Science and Technology Beijing, Beijing, China
}

\begin{abstract}
Chronic diseasesaccount for most of all deaths in developed countries and are increasing rampantly in newly industrialized nations. Physical exercise and sedentary behavior are risk factors for the major chronic diseases which increase rampantly in newly industrialized nations. In this paper, time trends of physical exercise and sedentary behavior in Chinese adults are examined and their five years effects on four chronic diseases morbidity are evaluated using logistic regression models with the CHNS data. Trend analyses show that physical exercise decreases while the sedentary behavior increases rapidly. Female, 18 30 years old, living urban and high education people are more likely to exercise during their leisure time. It also can be concluded that moderate physical exercise benefit one's blood pressure and prolonged sedentary increases the risk of suffering from myocardial infarction and apoplexy.
\end{abstract}

Keywords: physical exercise; sedentary; chronic diseases; risk factor

\section{INTRODUCTION}

Chronic diseases, also known as non-communicable diseases (NCDs), including cardiovascular diseases (such as heart attacks and stroke), cancers, type 2 diabetes, chronic respiratory diseases and diabetes are long-lasting and slow-progressing condition that can be controlled but not cured. They account for most of all deaths in developed countries and are increasing rampantly in newly industrialized nations (Daar et al., 2007). Experiencing rapid urbanization and socioeconomic growth, China bears a growing heave burden of morbidity and mortality of chronic diseases which are $22 \%$ and $85 \%$ respectively by 2014 according to the new data (Beaglehole, 2003 and Ghaffar, 2003). The study of the prevention and control of the chronic diseases is significant not just for individuals but for the whole aging society (Yang et al., 2008).

Unhealthy lifestyles are believed to be strongly correlated to chronic diseases.Lack of exercise andsedentaryarecommonphenomena in the modern society. Their effects on the chronic diseases have been studied by many researches (see Bernstein, 1994, Eriksson, 1991, Wannamethee, 1998, Thorp, 2011 and Owen, 2010). However, there is limited research about Chinese adult and most of them focus on the effect on certain disease mortality (Brummett, 2003 and Warren, 2010) or biological indicators (Owen, 2010 and Wood, 1983) rather than the morbidity.Some studies found that physical activity level of Chinese have been descending since 1990s and this trend should largely contribute to the urbanization of China and technology advancements (Ng, 2009 and Monda, 2008). In the meantime, the absence of an integrated program for $\mathrm{PE}$ in China highlights the significance of conducting

*Corresponding author: weiqing7@126.com further study on the PA and sedentary patterns and their effects on chronic diseases.

This study attempts to address these gaps by studying the effects of physical exercise and sedentary behavior on chronic diseases in a wide sample of Chinese adults aged 18-80. Using data from the China Health and Nutrition Survey (CHNS), we explore the time trend of physical exercise and sedentary behavior of a Chinese population followed by a 12 years period and discuss their effect on four chronic diseases morbidity with the 2004-2009 cohort.

\section{METHODS}

\subsection{Study population}

China Health and Nutrition Survey (CHNS), an international collaborative project between the Carolina Population Center at the University of North Carolina at Chapel Hill and the National Institute of Nutrition and Food Safety at the Chinese Center for Disease Control and Prevention (Yan et al., 2012), is an ongoing international collaborative longitudinal survey initially conducted in 1989 and subsequently surveyed every 2-4 years using structured questionnaires by trained field staff to a sample of about 4,400 households with a total of 26,000 individuals in 9 provinces that vary substantially in geography, economic development, public resources and health indicators[14] Additional CHNS details are available in previous publications (Dearth-Wesley, 2012, Fang, 2011 and Popkin, 2010)

Nevertheless, the survey is not national representative. Nine provinces sample can't reflex all twenty-threeprovinces, fourmunicipalities, fiveautonomous regions and twospecial administrative areas situation 
Table 1. Descriptive statistics* of individual level variables for adult Chinese men and women from five waves of the China Health and Nutrition Survey

\begin{tabular}{llllll}
\hline variables & 1997 & 2000 & 2004 & 2006 & 2009 \\
\hline No. of participants & 9191 & 7817 & 9259 & 9190 & 9541 \\
Age(year) & 41.8 & 43.5 & 47.2 & 48.5 & 49.3 \\
Male(\%) & 49.9 & 48.6 & 48.5 & 47.9 & 48.4 \\
Urban(\%) & 33.1 & 31.7 & 34.3 & 33.6 & 33.9 \\
Education(grade) & 1.5 & 1.7 & 1.8 & 1.8 & 1.8 \\
\hline
\end{tabular}

* means are calculated for numerical variables and percentages are calculated for categorical variables.

of China. The CHNS, however, is designed to monitor nationwide trends and provinces and communities of different levels are sampled, plus the non-participation rate is very low, so we are confident that the CHNS sample can provide good insight into the PE, sedentary behavior and chronic diseases relationship in China.

We use all available data from adults aged 18-80 years surveyed in 1997, 2000, 2004, 2006 and 2009. Records before 1997 are eliminated due to the lack of PE variables. Whereas, all data before 2004 survey year are excluded when sedentary behavior analysis is done. Individual missing data for critical variables are excluded $(4.75 \%)$, as are the disabled men and pregnant women (0.90\%). Eventually, 44997 observations are retained for the physical exercise trend analysis and 27989 for the sedentary behavior trend analysis. The effect of physical exercise and sedentary behavior on chronic diseases is examined by using 2004 cohort which contains around 5900 individuals.

\subsection{Study variables}

Chronic diseases variables: Four kind of chronic diseases information are collected by trained workers through posing question such as "has a doctor ever told you that you suffer from high blood pressure/diabetes/myocardial infarction/ apoplexy?". The effect on every disease is conducted separately.

Physical exercise variables: Respondents are asked whether they participate in the sixcategories PE, which are martial arts (Kung Fu, Tai Chi, etc.), gymnastics/dancing/acrobatics, track and field (running, etc.)/swimming, soccer/basketball/volleyball, badminton/tennis and other sports, one by one, and if the answer is "yes", their weekly minutes spent on them are recorded, otherwise, move on to the next sports category. The PE variables are processed to two forms, i) whether participant in any PE or not, ii) total hours per week spend on PE. Implausible high values are replaced with plausible maximum values.

Sedentary behavior variables: As with physical exercise, sedentary behaviors are obtained by raising questions like "do you participate in watching TV, surfing Internet, playing computer games or reading activities", and if the answer is "yes", then the average time spent on them every week is asked immediately. Finally, total hours per week spend on sedentary activities are calculated.

\subsection{Statistical analysis}

For descriptive analysis, percentages are calculated for categorical variables, while means are calculated for continuous variables. Table 1 lists statistical descriptive result of fivevariables for every wave, from which it can be concluded that the mean age and income of the study population increased. Logistic regression models are used to investigate the relationship of physical exercise, sedentary behavior and chronic diseases, adjusting for gender, age, education and residence and so on. The Logistic regression model is adopted because the response variable, namely whether one have a chronic disease or not in 2009 , is categorical. All statistical analyses are conducted using $\mathrm{R}$ version 3.0.2.

\section{RESULTS}

3.1 Time trends analysis of physical exercise and sedentary behavior in Chinese men and women

In this section, the time trend of the proportion of Chinese adults taking physical exercise and sedentary behavior in are examined from multi-lever and multi-angle. Physical exercise trend analysis use data from 1997 to 2009 while sedentary behavior trend analysis use data from 2004 because sedentary activities are not asked before then.

Figure 1 manifests that the physical exercise proportion has a different trend with previous physical activity study. The proportion of physical exercise is $16 \%$ in 1997 wave for Chinese men and decrease since then while the overall trend of the Chinese women shows an upward tendency and reaches a peak value of $10.3 \%$ in 2006. In 1997, the PE proportion of Chinese men was more than twice of that of the women, but the subsequent opposite trends of two genders narrow that gap down. One possible explanation for this phenomenon is the aging of Chinese population and prevalence of square dance in China Dama. The consistent but temporary increase of both male and female in 2006 probably owe to the 2008 Olympic Games which was going to be hold in Beijing and resulting the national fitness wave. What we most need concern is the excessive low proportion of physical exercise among Chinese adults. 


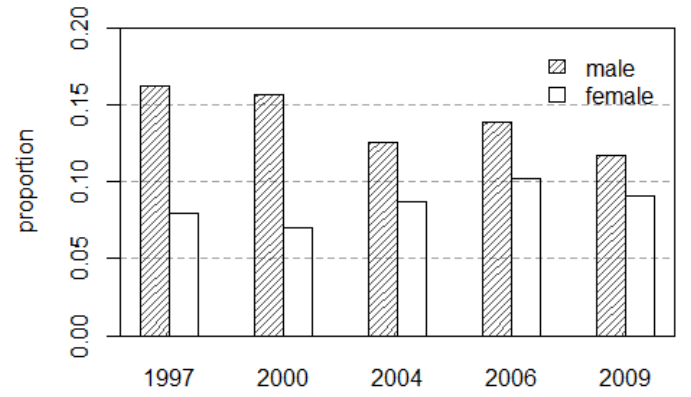

Figure 1. Physical exercise proportion of Chinese men and women in 1997-2009

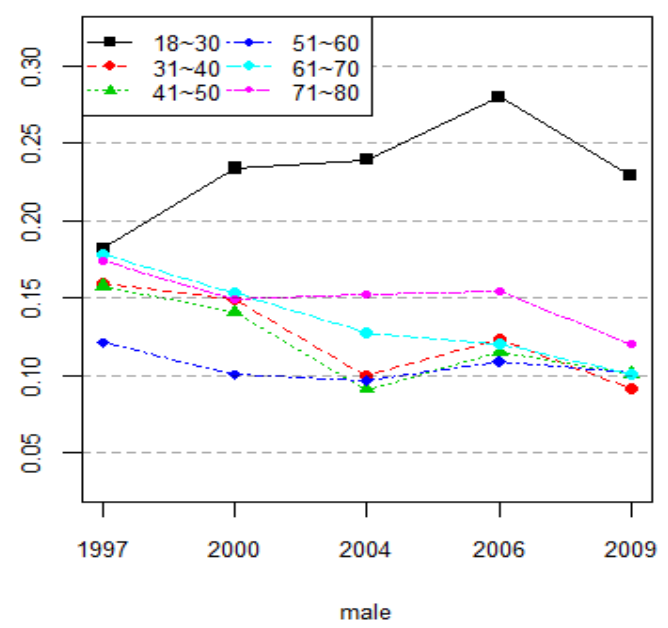

(a)



(b)

Figure 2. Time trends of PE proportion in different age groups for Chinese male and female in 1997-2009.
Since the complete different trends between Chinese men and women displayed, the following analysis has examined the proportion trends on different age, residence and education levels are all conducted separately for men and women (Figures 2 4). Consistent with above conclusion, figure 2 shows male has larger proportions than female in most waves and age groups, what's more, the proportion declines for male but increases inconspicuously for female. It also reveals that young adult under the age of thirty exercise most, followed by $61 \sim 80$ years old people, and then the 31 60 age group. This order is extremely evident for male and could be explained by the working age of Chinese adults. When people are young, they exercise much, and then decline with age to manage the family-work conflict, but after they retire, some of them have time to take physical exercise again.

In addition to age, residence and education also have relationship with physical exercise. It can be seen from Figure 3 that people live in urban have a higher proportion than those in rural for both genders. The female's proportion is still lower than male's and stay around 5\% in rural. Reluctant to admit, but the fact is that with China's economic development and wealth gap widens, Chinese farmers endure tremendous pressure and have a low quality of life. Besides, the tiring physical work in agriculture contributes to the lacking of exercise of the rural people, but Figure 4 also gives an explanation to some extent. In Figure 4 , $0 \sim 5$ represent the education level that every individual has from low to high are none, primary school, lower middle school, upper middle school, technical or vocational and university or above, respectively. The higher education level is, the greater the PE proportion is. In 2009, the PE proportion of male and female with university or above education level are $36 \%$ and $28 \%$, far exceed the average $11 \%$ and $9 \%$. Generally, people with high education level have more adequate awareness of health life style such as sufficient physical exercise, and there is relatively less manual labor during work.

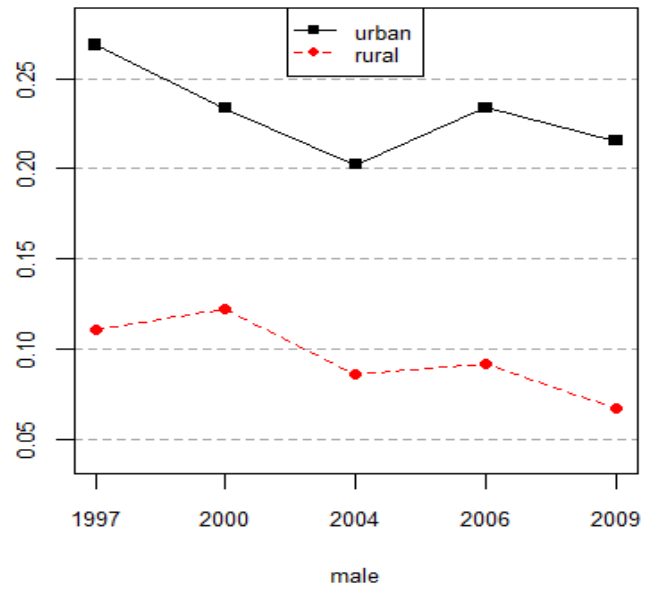

(a) 
MATEC Web of Conferences

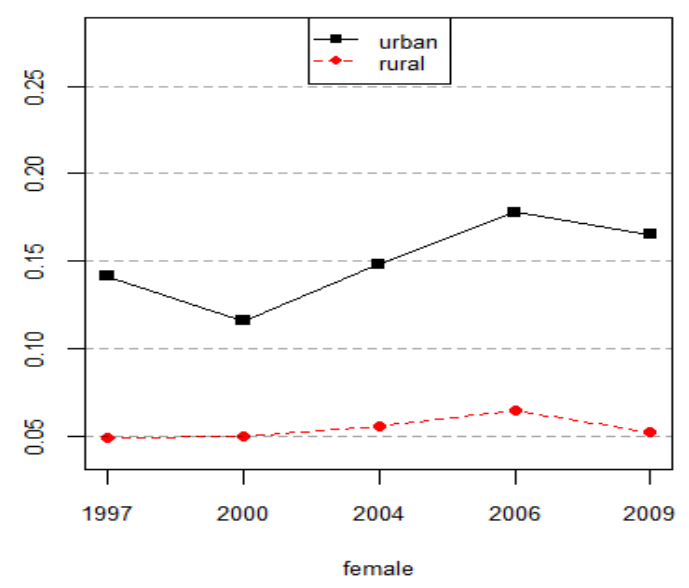

(b)

Figure 3. Time trends of PE proportion of different residence for Chinese male and female in 1997-2009.

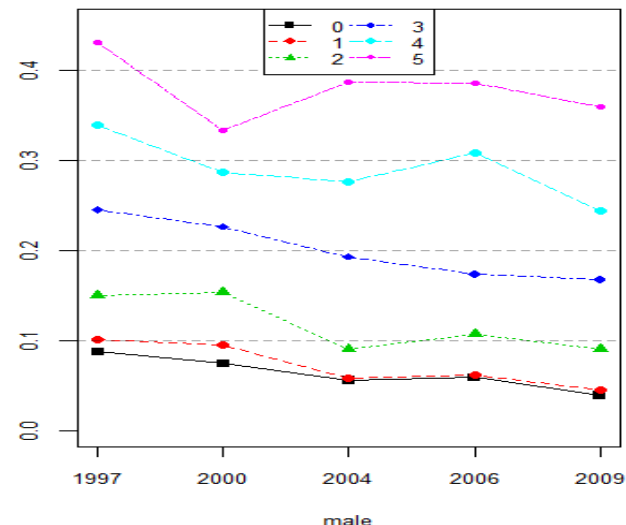

(a)

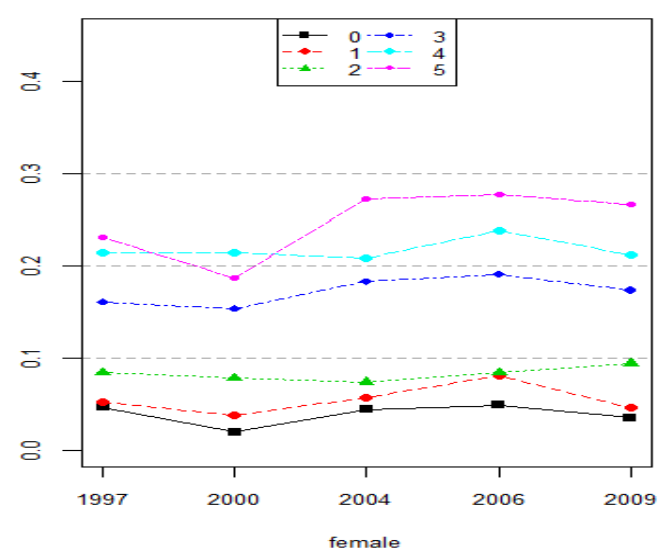

(b)

Figure 4. Time trends of PE proportion with different education level for Chinese male and female in 1997-2009
Figure 5 shows that while trend of PE time every week decrease among Chinese male from 2004 to 2009 , the sedentary behavior trends increase for both genders at the same period. The time of physical exercise is extremely low because of the small proportion of getting exercise among the population. From 2004 to 2009 , the mean time spent on sedentary behavior of Chinese male and female increased around 12\%. Mean hours spent on sedentary activities per week were 18 times the hours spent on physical exercise for male in 2009 and 25 times for female.

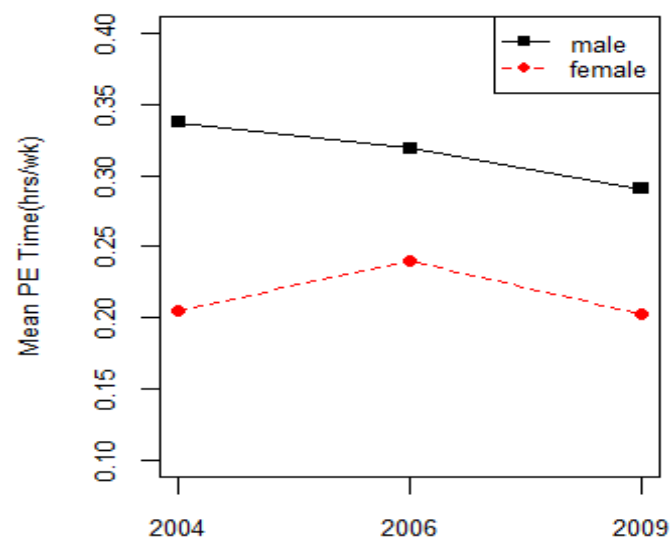

(a)

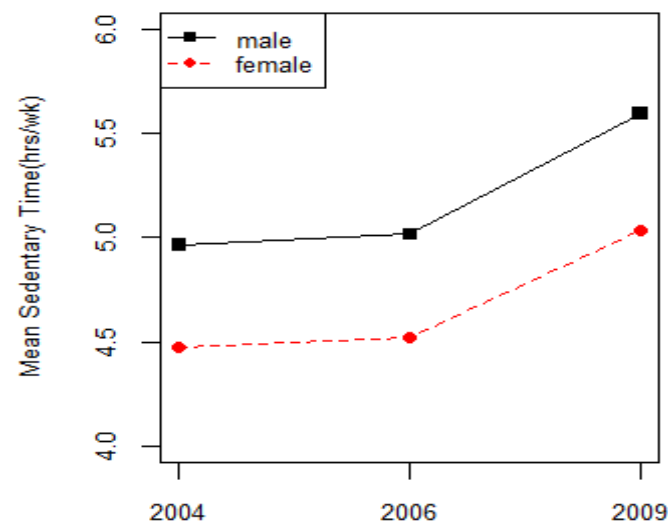

(b)

Figure 5. Mean hours per week of physical exercise and sedentary behavior in 2004-2009 of Chinese adults

\subsection{Effect of physical exercise and sedentary behav- ior on chronic diseases morbidity}

Four regression models are conducted to evaluate the effect of physical exercise and sedentary behavior on each disease morbidity. Respondents without one certain disease in 2004 survey are selected into the logistic regression model, and corresponding disease condition ( 0 or 1$)$ in 2009 as the response variable. Age, gender, residence, education level and baseline BMI, smoke condition are included in the models as 
ICETA 2015

Table 2. Coefficients of logistic regression models for four chronic diseases

\begin{tabular}{lllll}
\hline Covariate & High blood pressure & Diabetes & Myocardial infarction & Apoplexy \\
\hline Age & $0.060^{*}$ & $0.039^{*}$ & $0.083^{*}$ & 0.073 \\
Male & -0.206 & $0.561^{*}$ & 0.093 & 0.291 \\
Urban & $0.261^{*}$ & $0.411^{*}$ & 0.158 & -0.347 \\
Education & 0.005 & 0.103 & 0.161 & -0.013 \\
BMI & $0.167^{*}$ & $0.232^{*}$ & $0.107^{*}$ & $0.168^{*}$ \\
Smoke & & & \\
Ever smoker & $0.459^{*}$ & 0.078 & 0.102 & $1.398^{*}$ \\
Smoker & 0.236 & -0.016 & -0.450 & $0.842^{*}$ \\
PE & & & & -0.833 \\
$(0,3]$ & $-0.470^{*}$ & 0.127 & -0840 & 0.471 \\
$(3,6]$ & $-0.882^{*}$ & 0.656 & 0.079 & -14.151 \\
$(6$, max $)$ & -1.097 & -15.838 & -13.220 & -0.441 \\
Sedentary & & & & -13.903 \\
$(0,14]$ & 0.047 & -0.383 & 1.040 & $1.502^{*}$ \\
$(14,21]$ & 0.591 & -14.580 & 1.543 & $2.733^{*}$ \\
$(21$, max $)$ & 0.445 & -0.153 & & \\
\hline
\end{tabular}

$*$ coefficient is statistical significant $(\mathrm{p}<0.05)$

control variables. PE time (hrs/wk) and sedentary time (hrs/wk) are converted from continuous to categorical variables for their effect may not be linear. Regression coefficients of four models are listed in Table 2.

It can be concluded from Table 2 that the BMI of baseline is significant risk factor of every chronic disease which means additional BMI increase the risk of having high blood pressure, diabetes, myocardial infarction and apoplexy in 5 years. The coefficient of age for high blood pressure, diabetes, myocardial infarction is also positive, so older people are more likely having these disease but not apoplexy. Whereas, smoking is one of the major risk factors for the apoplexy. Coefficient of urban denotes that urban residents have higher likelihood of suffering from high blood pressure and diabetes disease than rural residents.

The first two coefficients of PE for high blood pressure is significant and negative, indicating that taking physical exercise less than three or six hours every week can greatly reduce the incidence of high blood pressure compared with doing no exercise. Sedentary exceed 21 hours per week increase the morbidity of myocardial infarction and apoplexy in the following 5 years. These findings suggest that even a very moderate amount of daily exercise, such as 30 minutes a day leading to health blood pressure and that adult who has more than 3 hours of sedentary time a day during leisure time is more likely to be attacked by myocardial infarction and apoplexy diseases.

\section{CONCLUSIONS}

Although this paper has provided insights into the association between physical exercise, sedentary behavior and chronic diseases, there are a number of limitations.

First, chronic disease morbidity is calculated according to the individuals' self-report disease, and there is high probability that respondents have suffered from some kind of chronic diseases but not examined by a doctor, hence the morbidity may not be accurate. Besides, four chronic diseases are far from enough to represent all chronic diseases. Second, sedentary behavior time estimated in this paper only contains activities during leisure time, and the sedentary time on work are not considered. As to the economic development, less labor work are needed, so the sedentary activities account for a large portion of most jobs and should be taken into consideration.

This paper only looks at what effects of physical exercise and sedentary behavior have on chronic diseases. Future work will include estimating a dynamic panel model that incorporates the community, household and more individual-level factors and relate them to changes over time.

\section{ACKNOWLEDGEMENT}

The research report here was partially supported by the NSFC (71420107023, 71231001 and 71301009), China Postdoctoral Science Foundation funded project (2013M530531), the Fundamental Research Funds for the Central Universities of China (FRF-MP-13-009A and FRF-TP-13-026A), and the MOE PhD supervisor fund (20120006110025).

This research uses data from China Health and $\mathrm{Nu}-$ trition Survey (CHNS). We thank the National Institute of Nutrition and Food Safety, China Center for Disease Control and Prevention, Carolina Population Center (5 R24 HD050924), the University of North Carolina at Chapel Hill, the NIH (R01-HD30880, DK056350, R24 HD050924, and R01-HD38700) and the Fogarty International Center, NIH for financial support for the CHNS data collection and analysis 


\section{MATEC Web of Conferences}

files from 1989 to 2011 and future surveys, and the China-Japan Friendship Hospital, Ministry of Health for support for CHNS 2009.

\section{REFERENCES}

[1] Beaglehole, R., \& Yach, D. 2003. Globalisation and the prevention and control of non-communicable disease: the neglected chronic diseases of adults. The Lancet, 362(9387): 903-908.

[2] Bernstein, L., Henderson, B. E., Hanisch, R., Sullivan-Halley, J., \& Ross, R. K. 1994. Physical exercise and reduced risk of breast cancer in young women. Journal of the National Cancer Institute, 86(18): 1403-1408.

[3] Brummett, B. H., Babyak, M. A., Siegler, I. C., Mark, D. B., Williams, R. B. \& Barefoot, J. C. 2003. Effect of smoking and sedentary behavior on the association between depressive symptoms and mortality from coronary heart disease. The American Journal of Cardiology, 92(5): 529-532.

[4] Daar, A. S., Singer, P. A., Persad, D. L., Pramming, S. K., Matthews, D. R., Beaglehole, R., ... \& Bell, J. 2007. Grand challenges in chronic non-communicable diseases. Nature, 450(7169): 494-496.

[5] Dearth-Wesley, T., Gordon-Larsen, P., Adair, L. S., Zhang, B., \& Popkin, B. M. 2012. Longitudinal, cross-cohort comparison of physical activity patterns in Chinese mothers and children. Int. J. Behav. Nutr. Phys. Act. 9: 39 .

[6] Eriksson, K. F., \& Lindgärde, F. 1991. Prevention of Type 2 (non-insulin-dependent) diabetes mellitus by diet and physical exercise the 6-year Malmö feasibility study. Diabetologia, 34(12): 891-898.

[7] Fang, H., \& Rizzo, J. A. 2011. Income inequality dynamics in rural China from 1991 to 2006: the role of alternative income sources. Applied Economics Letters, 18(14): 1307-1310.

[8] Ghaffar, A., Reddy, K. S., \& Singhi, M. 2004. Burden of non-communicable diseases in South Asia. $B M J$, 328(7443): 807-810.

[9] Monda, K. L., Adair, L. S., Zhai, F., \& Popkin, B. M. 2008. Longitudinal relationships between occupational and domestic physical activity patterns and body weight in China. European Journal of Clinical Nutrition, 62(11): 1318-1325.

[10]Ng, S. W., Norton, E. C., \& Popkin, B. M. 2009. Why have physical activity levels declined among Chinese adults? Findings from the 1991-2006 China health and nutrition surveys. Social Science \& Medicine, 68(7): 1305-1314.

[11] Owen, N., Healy, G. N., Matthews, C. E., \& Dunstan, D. W. 2010. Too much sitting: the population-health science of sedentary behavior. Exercise and Sport Sciences Reviews, 38(3): 105.

[12]Popkin, B. M., Du, S., Zhai, F., \& Zhang, B. 2010. Cohort Profile: The China Health and Nutrition Survey-monitoring and understanding socio-economic and health change in China, 1989-2011. International Journal of Epidemiology, 39(6): 1435-1440.
[13]Thorp, A. A., Owen, N., Neuhaus, M., \& Dunstan, D. W. 2011. Sedentary behaviors and subsequent health outcomes in adults: a systematic review of longitudinal studies, 1996-2011. American Journal of Preventive Medicine, 41(2): 207-215.

[14] Wannamethee, S. G., Shaper, A. G., \& Walker, M. 1998. Changes in physical activity, mortality, and incidence of coronary heart disease in older men. The Lancet, 351(9116): 1603-1608.

[15] Warren, T. Y., Barry, V., Hooker, S. P., Sui, X., Church, T. S., \& Blair, S. N. 2010. Sedentary behaviors increase risk of cardiovascular disease mortality in men. Medicine and Science in Sports and Exercise, 42(5): 879.

[16]Wood, P. D., Haskell, W. L., Blair, S. N., Williams, P. T., Krauss, R. M., Lindgren, F. T., ... \& Farquhar, J. W. 1983. Increased exercise level and plasma lipoprotein concentrations: a one-year, randomized, controlled study in sedentary, middle-aged men. Metabolism, 32(1): 31-39.

[17]Yan, S., Li, J., Li, S., Zhang, B., Du, S., Gordon Larsen, P., \& Popkin, B. 2012. The expanding burden of cardiometabolic risk in China: the China Health and $\mathrm{Nu}-$ trition Survey. Obesity Reviews, 13(9): 810-821.

[18] Yang, G., Kong, L., Zhao, W., Wan, X., Zhai, Y., Chen, L. C., \& Koplan, J. P. 2008. Emergence of chronic non-communicable diseases in China. The Lancet, 372(9650): 1697-1705. 